\title{
Errata
}

\section{Erratum: Coherence in singlet-exciton motion in anthracene crystals [Phys. Rev. B 31, 2430 (1985)]}

\author{
V. M. Kenkre and D. Schmid
}

The numbers quoted for the matrix elements of the intersite-transfer interactions were given in $\mathrm{Hz}$ instead of $\mathrm{rad} / \mathrm{s}$. For instance, the appropriate value of $V$ for singlet excitons in anthracene is $10^{13} \mathrm{~s}^{-1}$ rather than $1.5 \times 10^{12} \mathrm{~Hz}$. The resulting changes are as follows. In Tables I and II, the values for $\alpha$ should be multiplied by $(2 \pi)^{2}$ and those for $\Lambda$ (and $\Lambda / a$ ) should be divided by $2 \pi$. In Figs. 2 and 3 , the time scales should be divided by $(2 \pi)^{2}$ and the assumed fringe spacings should be divided by $2 \pi$. Thus, $\Lambda$ for singlet excitons at $1.8 \mathrm{~K}$ in pure anthracene crystals should be concluded to be $\geq 148 \mathrm{~nm}$ rather than $\geq 930 \mathrm{~nm}$. All the major conclusions drawn in the paper are unaffected by this change. In particular, the experiments analyzed do show singlet-exciton motion in pure anthracene at low temperatures to be highly coherent. However, it is unlikely that oscillations in grating signals can be observed in anthracene at the present state of experimentation. We are grateful to Professor V. Ern for pointing out this error to us.

In Eq. (5), there is an omission: The integral should be multiplied by $\alpha$.

\section{Erratum: Photoemission study of the hydrogenation of the intermetallic compounds $\mathrm{YFe}_{3}$ and $\mathrm{YFe}_{2}$ [Phys. Rev. B 31, 6167 (1985)]}

\section{H. Höchst, E. Colavita, and K. H. J. Buschow}

The annealing temperature quoted in the caption of Fig. 1 should read $800 \mathrm{~K}$ instead of $300 \mathrm{~K}$. The Ar $3 p$ emission could be removed at $600 \mathrm{~K}$; however, samples have been finally annealed to $800 \mathrm{~K}$. The crystal structure of $\mathrm{YFe}_{2}$ should read $\mathrm{MgCu}_{2}$ and not $\mathrm{HgCu}_{2}$.

We thank L. Schlapbach for making us aware of the misprints and apologize to the reader for any inconvenience.

\section{Erratum: Electron-phonon interactions and charge-density-wave formations in strong magnetic fields \\ [Phys. Rev. B 33, 5461 (1986)]}

\author{
Olle Heinonen and Radi A. Al-Jishi
}

It has been brought to our attention that Yorke and Bardasis ${ }^{1}$ have presented ideas very similar to those in our article. We are greatly indebted to Dr. A. Bardasis for making us aware of their work.

${ }^{1}$ E. D. Yorke and A. Bardasis, Solid State Commun. 32, 1251

(1979). 